\title{
Curbing Enthusiasm about Grounding*
}

\author{
JASON TURNER
}

January 10, 2017

Metaphysics in the high old style is back in fashion, and this season's favorite accessory is a relation philosophers are pleased to call metaphysical grounding. By 'grounding', the fashionistas do not intend merely the venerable project of trying to figure out how the ordinary appearances are settled by ultimate reality. They mean, instead, a very particular sort of metaphysical relation (or something like a relation) used to tie together the fundamental with the nonfundamental - a kind of priority relation that structures the world.

Some stodgier critics have objected that metaphysics' newfound enthusiasm for grounding is misguided, because the notion is in fact unintelligible: Talk of the alleged relation makes no sense, and metaphysicians have simply fooled themselves into thinking otherwise. I do not share this diagnosis - as far as I can see, talk of the relation makes perfect sense. But I too want to curb the discipline's grounding-based enthusiasm. The enthusiasm has led many to make wild claims about grounding's virtues, such as that we can use it to shield entities from Ockham's razor. And it has led many to embark on grand grounding-theoretic projects, such as the search for its 'logic'. I think many of these claims unfounded and projects unpromising: Grounding's marketing campaign has made promises which, I will argue, it cannot deliver.

\section{Two Notions of Grounding}

What is grounding? Most of its fans deny that it can be defined by any more basic terms. Rather, they explicate it by pointing to alleged examples of the phenomenon. If you can grasp the pheonmenon in the examples, and see how it could extend to further cases, you'e got the notion.

The main examples, it seems to me, come in two varieties. Here are some examples of the first variety:

\section{Variety One}

- Sets are grounded by their members. (E.g., $\{$ Jason $\}$ is grounded by me.)

- Wholes are grounded by their parts. (E.g., I am grounded by my head, torso, arms, legs, etc.)

- True propositions are grounded in how the world is. (E.g., the truth of the proposition that grass is green is grounded in grass's being green.)

*Many thanks to Ross Cameron, Jack Woods, and Shamik Dasgupta for helpful comments and conversation. 
And here are some examples of the second variety:

\section{Variety Two}

- True disjunctions are grounded in true disjuncts. (E.g., that grass is either red or blue is grounded in grass's being blue.)

- Existential truths are grounded in their instances. (E.g., that someone is standing is grounded by my standing.)

- Determinable states are grounded in their determinates. (E.g., that the patch is red is grounded by the patch's being scarlet.)

On the basis of our grasp of some combination of examples from these two lists (or similar sorts of examples), we are supposed to conclude that there is a single phenomenon, grounding, that we should take seriously.

It seems to me that there are in fact two very different phenomena being picked out here. There may be some family resemblance; perhaps both are a distinctive sort of metaphysical explanation. But their differences seem important enough to warrant keeping the two notions separate.

Some prima facie evidence for the difference comes by considering how these claims interact with counterfactuals. If we have a claim of the form 'It's being the case that $Q$ is grounded in it's being the case that $P^{\prime}$, let it's missing-ground counterfactual be the counterfactual, 'If $P$ hadn't been the case, $Q$ wouldn't have been the case'. Each of the first three grounding claims pairs naturally with its missing-ground counterfactual; that is, it is fairly easy to hear each of these as true:

- $\{$ Jason $\}$ is grounded in me; if I hadn't existed, $\{$ Jason $\}$ wouldn't have existed.

- I am grounded in my head, torso, arms, legs, etc.; if they hadn't existed, I wouldn't have existed.

- The truth of the proposition that grass is green is grounded in grass's being green; if grass hadn't been green, the proposition wouldn't have been true.

On the other hand, the pairings of the claims in the second list with their missing-ground counterfactuals are far less natural:

- Grass's being green or blue is grounded in its being green; if it hadn't been green, it wouldn't have been green or blue.

- That someone is standing is grounded by my standing; if I hadn't been standing, nobody would have been standing.

- That the patch is red is grounded in the patch's being scarlet; if the patch hadn't been scarlet, it wouldn't have been red. 
Uses of 'grounds' in the first list seem to put us in a position where we count possibilities without the grounds as counterfactually 'closer' than possibilities with them - even in cases where, as it is with me and my parts, we generally think I could have existed with different parts. Uses of 'grounds' in the second list don't seem to affect our judgments about counterfactual closeness. Learning that grass's green-or-blue-ness is grounded in its greenness doesn't make me count worlds where grass is blue as 'further away' than ones where, for instance, it is red.

Before going on, let's regiment our talk a little bit. Grounding's friends often (though not always) think of it as a relation holding between facts. I would prefer to be more neutral, though. Since facts are a sort of reification of sentences, we can de-reify the facts by replacing them with the sentences meant to pick them out. If we do this, we will have to trade in a 'grounding' predicate, meant to express a relation, for a grounding operator. For now, we'll use two: One $\left({ }^{\prime}{ }^{\prime}\right)$ will be used for the sort of 'grounding' in the first list; the other (' $<$ ') will be used for the sort of 'grounding' in the second. Then our lists become:

\section{Variety One}

- $\exists x(x=\{$ Jason $\}) \longleftarrow \exists x(x=$ Jason $)$.

- $\exists x(x=$ Jason $) \longleftrightarrow \exists w, x, y, z(w=$ Head \& $x=$ Torso \& $y=$ Arms $\& z=$ Legs \&__ ). [Fill in the blank with whatever conditions you think are required for Head, Torso, Arms, and Legs to compose someone.]

- The proposition that grass is green is true $\longleftarrow$ grass is green.

\section{Variety Two}

- Grass is either green or blue < grass is green.

- $\exists x(x$ is standing $)<$ Jason is standing.

- The patch is red $<$ the patch is scarlet.

So what is going on in these cases? Start with variety two. In examples such as these, the right-hand side seems to be making a more specific claim than the left-hand side - intuitively, it says what the left-hand side says, and more besides. We might put the point by saying that the information on the 'grounded' side is contained in the information on the grounding side. We might find that overly representational, though. Here are a few other ways to try to get at the thought: (i) The state described by the right hand side is something like an instance of the one on the left. (ii) The right-hand-side witnesses the left-hand side. (iii) The right-hand-side is a way for the left-hand side to be realized. ${ }^{1}$

\footnotetext{
${ }^{1}$ We might also want to describe it in truthmaking terms: Grass's being green is what makes it true that grass is either green or blue. (Cf. Cameron 2015: 114-117) I have no principled objection to such a description, but worry it isn't fine-grained enough to capture the distinction
} 
Examples of variety two naturally fit with a number of other things we might want to say. For instance, we might say 'In order for God to make grass either green or blue, she only needs to make grass green'. Or we might say 'that grass is in fact green or blue is nothing over and above the fact that grass is green.' There's nothing particularly tendentious about these claims, given that the greenness of grass just is one way for it to be green or blue. Once we imagine grass being green, it is hard to imagine what else we would have to add to the situation in order to also imagine that grass is either green or blue. It seems obvious that we are already there.

These sorts of claims are much less compelling when made about the Variety One examples. I realize that people sometimes do make these claims; I just don't see why they should. Once God has made me, must God do anything else to get my singleton? Well, yes: God must make sure that my existence suffices for the existence of my singleton. Is the existence of my singleton 'nothing over and above' my existence? It is hard to see how. After all, my singleton isn't 'nothing over and above' me - if it was, it would be me. And it is simple enough to imagine me without my singleton. Why? Because the information in '\{Jason\} exists' is not contained in 'Jason exists': The former adds extra set-theoretic information. Nor is my existence a 'way' for the existence of my singleton to be realized. Nor is my existence a witness to the existence of my singleton. My singleton's existence isn't some less-specific state of which my existence is a more-specific version; nor are they just the same state re-described.

The natural reading of the Variety One sentences is different: They seem to be saying that the grounded things (sets, wholes, propositional truth) metaphysically depend on their grounds. The relation between the grounds in the Variety One examples and what they ground is more like a metaphysical correlate of causation: my singleton is grounded in me because it depends on me for its existence; the proposition that grass is green depends on grass's being green for its truth. And just as natural causation requires natural laws, metaphysical causation requires metaphysical laws. That is why it is easy enough to imagine me without my singleton; to imagine me is not yet to imagine the metaphysical law connecting me with my singleton.

Call the relation in the Variety One examples, when understood this way, dependence-grounding; we will use ' $\longleftarrow$ ' for this relation. And call the Variety Two examples' relation realization-grounding; ' $<$ ' gets used for it. These are two very different conceptions of importantly different relations, and we would do well to keep them separate. ${ }^{2}$

between Variety One and Variety Two examples. Are the existence of my parts what makes it true that I exist? Maybe - but if so, they do it in a very different way than grass's greenness makes is true that grass is green or blue, and it is the difference in these ways that I want to highlight here.

${ }^{2}$ I am not alone in thinking that 'grounding' talk is unfortunately ambiguous; Jessica Wilson (2014) and Kathrin Koslicki (2015) argue for this as well. But the disambiguations I identify aren't obviously the same ones they do. Perhaps I am agreeing with Wilson about one of the disambiguations ('realization-grounding') while taking her other disambiguations and lumping 
(Some who recognize this distinction insist that only one or the other of these two relations is 'really' grounding, the other being something else entirely. Others insist that 'real' grounding is something like the disjunction of these two relations (or maybe its transitive closure), or a more general 'metaphysical explanation' of which these two relations are species. These disputes strikes me as entirely terminological, and I cannot imagine what could settle them short of linguistic fiat. There are two importantly different relations $(<$ and $\longleftarrow$ ) which we need to recognize and which figure in various authors' attempts to motivate 'grounding' in metaphysics. What's ultimately at stake is the features of these two relations, whatever we decide to call them.)

I have divided up the varieties by what I think are the most natural readings of the claims, but I do not say they are the only ones. If we understand the patch's redness as being realization-grounded in the patch's scarletness, we are most likely thinking of determinates as something broadly akin to disjunctions of determinates; to be red just is to have one of red's determinables. Jessica Wilson (2012) has argued for a determinable/determinate distinction that breaks this close tie. On her view, it is possible for something to have a determinable without having any of its determinates. Someone who held a view like this may think that, when the patch is scarlet, it 'forces' the patch to instantiate redness as well, and give voice to that by saying 'the patch's being red is grounded in its being scarlet'. But this would be a kind of dependence-grounding; the scartlet-ness isn't a witness of its redness but rather something like a 'metaphysical cause.'

By the same token, certain views might make us understand some of the the Variety One examples in the realization-grounding way. Consider, for example, a particular kind of neo-Fregean who thinks that set-theoretic talk, like number talk, can be introduced by stipulation. The stipulation doesn't create new things — sets - nor does it serve to describe some self-standing realm of objects than we had been ignoring before making the stipulations. Rather, the stipulations simply assign old set-free contents to new, set-theoretic-using sentences. Once the stipulations are introduced, for instance, ' $\{$ Jason $\}$ exists' just becomes a fancy way of saying that Jason exists. If that is what ' $\{$ Jason\} exists' really means, then the informational content of ' $\exists x(\{x\} \text { exists })^{\prime}$ is precisely the same as that of ' $\exists x$ ( $x$ exists $)$ ', and so would be contained in 'Jason exists'. Such a theorist could then say that the existence of some set is grounded in my existence. Since my existence is one way for the state expressed by ' $\exists x(\{x\} \text { exists })^{\prime}$ to obtain, the theorist could understand this as a case of realization-grounding.

Or consider a deflationist about truth. According to deflationism, 'true' is a mere expressive device: 'It is true that grass is green' is simply a longwinded

them all together into a single glob ('dependence-grounding'). If so, then I think this speaks to a deeper disagreement between us, as I think it is useful to keep this glob as a glob and not split it into parthood, set-membership, etc., as she does. See also Barnes 2012 for a related distinction; I suspect her dependence is closely allied with my 'dependence-grounding', but I'm less sure that her contrasting 'fundamental/derivative' talk patterns with my 'realization-grounding.' 
way of saying that grass is green. Such a deflationist could also be a deflationist about propositions: 'The proposition that grass is green is true' could be just an even longer-winded way of saying that grass is green. This deflationist doesn't think that reality comes pre-equipped with two kinds of thing - grass and propositions - and that one of these things 'tracks' what the other is doing. Rather, reality just contains the grass. Proposition-talk is just that: talk. On a view like this, 'The proposition that grass green is true' has the same informational content as 'Grass is green', and for similar reasons these can be used to cook up a true realization-grounding claim.

On either of these views, we may be tempted to say something even stronger:

(1) $\exists x(x=\{$ Jason $\})<\exists x(x=$ Jason $)$.

(2) The proposition that grass is green is true $<$ grass is green.

And we are tempted to make the same sorts of noises about these claims that we made about other <-using claims. Once we realize that, for the neo-Fregean, saying that my singleton exists is just a fancy way to say that I exist, it makes more sense to say that my singleton really is nothing over and above me. And since the deflationist thinks that the truth of the proposition that grass is green is nothing more or less than grass's being green, we of course think that once God makes grass green there is nothing left for her to do in order to make the proposition true. But in both cases that's because, in an important sense, there aren't really sets or propositions — just talk about them that's licensed by a deeper set- or proposition-free reality.

Some friends of grounding, I suspect, would not like the neo-Fregean or deflationist to endorse (1) or (2). They would object that the irreflexivity of grounding - that nothing can ground itself — tells against (1) and (2) when the sentences on each side of the ' $<$ ' have the same informational content. That is, they take the irreflexivity of grounding to mean that, if $P$ and $Q$ have the same informational content, then $P \nless Q$.

But does it really mean this? There are reasons to think not. First, most treatments of realization-grounding make it multigrade: claims such as $S<P, Q, R$ are intelligible. An information-theoretic gloss might understand this as saying the information in $S$ is collectively contained in $P, Q$, and $R$; a less representational gloss might understand it as saying that the collective obtaining of the $P$ - $Q$ - and $R$-states are one way for $S$ to obtain, or that $P, Q$, and $R$ collectively witness $S$. The reason for the multigrade treatment is to make room for the grounding of conjunctions; most 'logics' of grounding accept

(CONJ) If $P \wedge Q$, then $P \wedge Q<P, Q$.

A second, commonly endorsed thesis is that of contraction:

(CONT) If $R<P, P$, then $R<P$. 
This principle says, roughly, that redundant grounds can be eliminated. This makes a lot of sense, on the realization-theoretic approach; after all, the collective information contained in $P, P$ is precisely the information contained in $P$. But it is very easy to use these to derive

(3) If $P$, then $P \wedge P<P$.

But $P \wedge P$ seems to obviously to describe the same state as $P$; these are different ways of describing the same underlying part of the world. If (3) is okay, I have a hard time seeing why, given the neo-Fregean and deflationist views, (1) and (2) would not be. ${ }^{3}$

Sometimes theorists insist that grounding hold between facts, and reify accordingly. (They will use a grounding predicate, $\overline{<}$, rather than a grounding operator, $<$.) Those who then insist on an asymmetry principle saying that, if $F<G$, then $F \neq G$ will be forced further to say that the fact that $P$ is distinct from the fact that $P \wedge P$. This strikes me as a bad place to end up. We posit facts to be the worldly correlates of propositions - chunks of reality that make propositional representations accurate. If the fact that I am human is a different chunk of reality than the fact that I am human and I am human, I no longer understand the point of having facts around. It is, to use the old phrase, buying a dog and doing the barking yourself. If our a priori reflection on grounding pushes us here, our a priori reflection is suspect.

Those who refuse to reify facts face no such problems. They can understand asymmetry as the schema $P \nless P$. So long as they remember that ' $<$ ' is hyperintensional, so that equivalent sentences (even logically equivalent sentences) cannot be substituted for each other, they run into no problems from (CONJ) and (CONT).

Those who reify can avoid the problems similarly. Instead of understanding asymmetry as saying that, if $F \overline{<}$, then $F \neq G$, they can simply understand it as the schema $F \nless F$. They must then remember that $\overline{<}$ creates an opaque context, where co-referring fact names cannot be substituted salva veritate.

Anyone who takes one of these two routes recognize the hyperintensionality of realization-grounding by a ban on freely substituting equivalents. If we insist on substitution, and we also insist on (CONJ) and (CONT), we have to find some other way to deal with the hyperintensionality. Those who distinguish the fact that $P$ from the fact that $P \wedge P$ do it by locating the hyperintensionaity in the ontology. This seems like exactly the wrong place to put it. The truth of (3) doesn't depend on each side of the grounding claim describing different chunks of reality, but on one side describing the same chunk as the other, but in a metaphysically better way.

\footnotetext{
${ }^{3}$ One way to accommodate both of these principles is by appeal to Kit Fine's (2009) distinction between strict and weak grounding, and hold that (CONJ) and (CONT) hold only in full generality for the weak notion. That is fine; if so, then let us accept (1) and (2) as true under the weak notion too, and be done with it. Correia (2010) suggests instead restricting (CONT) to cases where $Q$ is not 'factually equivalent' to $P$. I see that this is possible, but I don't see why it is well-motivated.
} 
This suggest that $<$ works more-or-less as follows: when $P<Q$, the state described by $Q$ must be a witness for, or a specific way to realize, the state that $P$, and when both describe the same state $Q$ 's presentation of it must do a strictly better job of reflecting reality's underlying structure. Since ' $\{$ Jason $\}$ exists' reflects reality's underlying structure as involving sets and the proposition that grass is green is true' reflects it as involving propositions, but on the respective theories reality's ultimate structure doesn't contain sets or propositions, (1) and (2) are vindicated for the respective theories.

One final point about these notions. We are supposed to find them intelligible thanks to the examples used to motivate them. Some insist they do not understand the examples, ${ }^{4}$ but I must confess that I think I do. Some philosophers go further, though, insisting that grounding must be understood as fundamental: it must be taken as both conceptually and metaphysically basic. I am not yet ready to acquiesce to that claim, and I intend to remain neutral on it here.

\section{ENTHUSIASM}

Fans of grounding have put it to work in several ways. One branch of the literature focuses on the logic of grounding. It attempts to cook up a priori principles that grounding must obey; the idea, presumably, is that theories which violate those principles can be ruled out of bounds. A separate branch of the literature uses grounding to make ontology easier: It holds that a grounded ontology is somehow less objectionable and easier to get along with than an ungrounded one, and recommends replacing venerable debates about whether there are suchand-such things with debates about whether such-and-such things are grounded or not. And third strand, common to both literatures, insists that we can understand the vexed notion of fundamentality in grounding-theoretic terms.

I think that each of these is, to a greater or lesser extent, a mistake, and I'm going to argue for that here. In some cases, the mistake is limited only when the job is being done by one sort of grounding rather than another; in other case, the mistake cuts across the different notions.

In §3 I explain the risks of thinking grounding the ultimate key to understanding fundamentality. I do not think I say anything new here, but we can all stand to be reminded of old truths sometimes. In §4 I raise several worries about the prospects for finding a single, once-and-for-all, logic of grounding. Finally, I turn to ontology. In $\S 5$, I argue that ugrounded entities are no 'free lunch,' despite pleading to the contrary, and in $\S 6$ I argue that if we focus our attention only on grounding-based theories, we miss important prospective solutions to pressing philosophical problems.

\footnotetext{
${ }^{4}$ E.g., Chris Daly (2012) and Thomas Hofweber (2009: 266-273).
} 


\section{Grounding and Fundamentality}

It has become popular, among friends of grounding, to identify the fundamental with that which is ungrounded. There is something to the idea; ungroundedness is certainly a kind of fundamentality. The risk is that the identification can mask certain other, equally useful, senses of fundamentality.

If 'fundamental' just means, always and everywhere, ungrounded, then (as Jessica Wilson (2014: 543-544) has pressed) we lose one particularly attractive way of understanding emergence. Elizabeth Barnes (2012) suggests that emergent properties are those which are both fundamental and grounded in (or metaphysically dependent upon) lower-level properties. If 'fundamental' means 'ungrounded', though, Barnes's proposal has it that emergent properties are both grounded and ungrounded. So there had better be a way to understand 'fundamental' that doesn't just mean 'ungrounded'.

One proposal stems from our distinction between realization- and dependence-grounding. Perhaps the emergent properties are those that are dependence-grounded in lower-level properties but not realization-grounded in such properties. If so, the distinction between two kinds of grounding leads to a distinction between two kinds of fundamentality.

But this understanding isn't mandatory. David Lewis (1983a) famously presented a picture of fundamentality that appealed to perfectly natural properties: properties that 'carve reality at its joints', that make objective distinctions between similarity and dissimilarity and perform a host of other important theoretical roles. Perhaps emergent properties, on Barnes's proposal, should be understood as perfectly natural properties that nonetheless are dependencegrounded in distributions of lower-level perfectly natural properties. If so, then there is a way to understand 'fundamental' which is entirely divorced from grounding considerations.

'Fundamental' is said in many ways. There can surely be no objection to thinking that one of these simply means 'ungrounded' (suitably disambiguated). The risk is in thinking that we have now discovered 'what fundamentality really is', or what we meant with our fundamentality-talk all along. To think this is to miss out on important theoretical options (such as Barnes's), and to court both misunderstanding and being misunderstood by those who use 'fundamental' in a different way.

\section{The Logic of Grounding}

Much ink has been spilled over the 'logic of grounding'. ${ }^{5}$ The idea comes in two parts. The first is that grounding should obey some general structural principles: it should be asymmetric and irreflexive, for instance, and quite possibly

\footnotetext{
${ }^{5} \mathrm{~A}$ small sample of that ink can be found in Fine 2009, deRosset 2014, Litland 2015, and Correia 2010.
} 
transitive as well. The second is that there should be some substantive constraints on how grounding interacts with logical notions. For instance, as alluded to earlier, grounding is supposed to satisfy

- If $P \vee Q$, then either $P \vee Q$ is grounded in $P$ or $P \vee Q$ is grounded in $Q$.

- If $P \wedge Q$, then $P \wedge Q$ is grounded in $P, Q$.

- If $\exists x F x$, then for some $a, \exists x F x$ is grounded in $F a$.

The idea is not merely the relatively benign one that, as it happens, grass's being green-or-blue is grounded in its being green. The idea is that every true disjunction must be grounded in a true disjunct. It is a general law of grounding, admitting of no exception.

The idea's first part strikes me as reasonable, although even the alleged asymmetry of grounding is controversial (cf. Barnes forthcoming), and I am not sure the controversy has been fully grappled with by grounding's logicians. I am more skeptical about the idea's second part, though: I doubt there are any such substantive constraints on grounding. For all I know, it may just happen that all true disjunctions are grounded in true disjuncts and so on. But I don't know that it's some sort of inviolable 'law of grounding', with such hold on us that theories which demur can be rejected out-of-hand.

The examples used to motivate the project line up best with grounding-asrealization, and I suspect that most grounding-logicians are in fact thinking of grounding in a way that more-or-less tracks this notion. But the project could in principle be undertaken on behalf of dependence-grounding, too. The source of my skepticism changes depending on which notion is at stake; I will outline both below.

\subsection{Preliminary Grumbling}

I have specific reasons to be skeptical about substantive constraints on grounding. Before I get to them, let me note a general worry about the way the project proceeds.

In literature concerned with the 'logic' of grounding, it isn't too difficult to find bold, confident assertions of some feature or other of the phenomenon in question. Consider a few examples:

... it seems quite clear that if there are disjunctive facts, then a disjunctive fact is grounded in its true disjuncts... For similar reasons, it seems clear that existential facts are grounded in their instances. (Rosen 2010: 117)

If $\Delta$ is a strict full ground for $A \wedge B(\Delta<A \wedge B)$, then, for some division of $\Delta$ into the parts $\Delta_{1}$ and $\Delta_{2}, \Delta_{1}$ and $\Delta_{2}$ are weak full grounds for $A$ and $B$ respectively. (Fine 2012: 64) 
It is implicit in this idea, first, that grounding relations are general, in the sense that what a property enables a thing to do does not differ among the various instances of that property, and second, that grounding relations are necessary, in the sense that what properly enables a thing to ground does not differ across possible worlds. (Audi 2012: 697)

I have to confess I find these bold pronouncements puzzling. 'Grounding' is a theoretical notion, and the only grip we really have on it is given to us by the cases that introduce it. I may understand just fine what's being said when someone says that grass's being blue or green is grounded in its being green. But that, all by itself, can't justify a universal law. What seems to happen is that we start with a few simple cases and then think, along with Fine (2012: 60), that 'it would be desirable, in any case, to have uniform rules of ground for the logical constants.' From this we make some grand sweeping claims about how grounding always interacts with disjunctions or whatever, and we're off to the races.

But why think that what we desire is true? A nice clean logic of causation would be nice, but I think by now most of us are resigned to the fact that we're never going to get one. Causation just isn't the kind of thing that is tightly constrained by logical principles. What justifies us in assuming that grounding is better off than causation here?

Nor do I find it helpful to stipulate that grounding is a special sort of 'metaphysical explanation.' (Fine 2012: 39, Dasgupta 2014: 3, Litland 2015: 483) A lot of things are metaphysical explanations, by any reasonable standard of 'metaphysical' and 'explanation': David Lewis's analysis of possibility in terms of disconnected spacetimes proposes a metaphysical explanation of possibility, and Hartry Field's Science Without Numbers project proposes a metaphysical explanation of the usefulness of mathematics. But I don't see how to faithfully translate either of those projects into a formally regimented 'grounding' notation, satisfying various logical principles.

Furthermore, our grip on 'metaphysical explanation' doesn't seem a whole lot firmer than the grip we get on any notion introduced by examples. Except for a general ban on circularity, there aren't a whole lot of obvious structural principles governing explanation. And the bold-faced insistence that 'identities can't explain' looks misguided. If I understand 'metaphysical explanation' at all, then a paradigm example of it comes from special relativity's explanation for why nothing can travel faster than light. The answer is: To travel faster than light is to be located on a trajectory that lies outside a light cone; but to travel just is to be located on a time-like trajectory, which is to say a trajectory that lies inside (or on the surface of) a light cone. To travel faster than light then requires the contradictory: traveling on a trajectory that is both within and not within a light cone. The explanation proceeds by pointing out identities between travel, on the one hand, and four-dimensional geometric features, on the other, and showing that once we unpack the identities the notion of traveling faster than 
light is contradictory. Identities form parts of metaphysical explanation, if that is anything like what we ordinarily think it is.

These grumblings don't give us direct reason to think that there are no substantial constraints on grounding. (You'll have to wait for the next section for that.) They are meant instead to sound a cautionary note. Knowing someone by sight doesn't give you insight into their inner lives. Likewise, recognizing grounding when you see it doesn't mean you really understand how, deep down, it works. A lot of claims can seem obvious when generalized from only a few cases, and the cases themselves can give us the wrong idea about just what grounding is all about. It is very, very unclear what justifies our beliefs about how grounding works, and in such an epistemically precarious position we ought to proceed with caution.

\subsection{Against Substantial Constraints}

\section{Dependence-Grounding}

As I've already indicated, I suspect that the project of finding substantial logical constraints on grounding makes a lot more sense if grounding as understood as realization rather than dependence. Rather than arguing for that directly, let me highlight an oddity that happens if we impose standard logic-of-grounding rules on dependence-grounding.

Start with an attractive view about identity. In the Tractatus, Wittgenstein says that identity is not a relation (5.5301-5.5303). What he seems to have in mind is this: Identity isn't anything that's added to an entity once it exists. Once you get an entity, you automatically get all its identity facts in the bargain. (Turner 2013)

Here's a weaker version of the same view: Once you get an entity, you get its self-identity fact in the bargain. My self-identity isn't something that 'floats free' of me; rather, my existence gives rise to my self-identity. This is a really attractive view, and the friend of dependence-grounding may want to accommodate it by saying that self-identity facts are grounded in existence facts. Thus we get

(4) Jason $=$ Jason $\longleftarrow \exists x(x=$ Jason $)$.

But one of the most compelling logic-of-grounding principles has is that existential facts are grounded in their instances, which gets us

(5) $\exists x(x=$ Jason $) \longleftarrow$ Jason $=$ Jason.

Grounding was supposed to be asymmetric, which is violated by (4) and (5)! Of course, the self-identity-is-grounded-in-existence view isn't mandatory. But it should at least be permissible - we should not rule it otherwise by treating (5) as sacrosanct. 
A friend of dependence-grounding might, following Jonathan Schaffer (2009), treat it as a relation between entities rather than a relation between (only) facts, or (as I have been treating it) something like a sentential operator. Then grounding will hold between individuals and facts both. This Schafferian can then grant the Tractarian thought by having the fact that I am self-identical be grounded in me, and having the fact that I exist be grounded in the fact that I am selfidentical. Then the 'logical' principle can be accommodated alongside the Tractarian thought.

I see that the move can be made, but I can't see why we would want to. If facts can depend on entities - if that's an idea that makes sense - then presumably the fact that I exist should depend directly on me. On the imagined Schafferian picture, it does depend on me, but only thanks to depending on my self-identity as a middleman. But why the detour? It doesn't seem independently motivated by the metaphysics itself, but rather a wrinkle to vindicate an otherwise-unmotivated 'logical' principle.

The claim that existential truths are grounded in their instances has some pull if by 'grounding' we are thinking of it in a realization or 'witnessing' way: instances of existential truths are witnesses of those truths themselves. If we are thinking of grounding as a kind of dependence, though, it seems much less compelling. I can't see any good sense in which my existence depends on my self-identity. As such, I can't see that there's any sensible 'logical' project for dependence-grounders. ${ }^{6}$

\section{Realization-Grounding}

The last example is less compelling if we think of grounding as realization; it's less tempting to think (as a realization-grounding translation of (4) would have it) that my existence is a witness of, or a way of realizing, my self-identity. But other examples raise similar worries.

Take, for instance, Argle from the Lewis's (1970) dialogue, "Holes". According to Argle, there are no holes, but "There is a hole in this piece of cheese," is true. That's because Argle endorses a fancy semantics according to which, when 'a hole' is attached to 'there is', it doesn't act like a quantifier but instead a predicate which means is perforated. ${ }^{7}$

Consider the claim that, for some entity $a$,

(6) There is a hole Argle's piece of cheese $<a$ is a hole in this piece of cheese.

\footnotetext{
${ }^{6}$ Fine (2012: 59-60) considers a related issue and concludes that existence claims should not be understood in terms of existential quantification. If that's right, then (4) could be given up for a variant where my self-identity is grounded in 'my existence' understood in this other (not yet specified) way. But my complaint is still essentially the same: Why should we give up a perfectly good understanding of existence claims just to satisfy some arbitrary 'logic-ofgrounding' demand?

${ }^{7}$ This is Argle's view at the beginning of the dialogue, anyway. He changes his tune at the end, but we will focus on Early Argle's songbook.
} 
This is a bizzare thing for an Argelian to endorse. (6) says that the state of there being a hole in Argle's cheese is realized, or witnessed, by some particular entity being the hole in Argle's cheese. But this is precisely what Argle denies. There is a hole in Argle's cheese, but this isn't witnessed by any entity at all. Rather, it is witnessed by the cheese having a particular perforated shape. As a result, if Argle is right, it existential truths aren't always grounded in their instances.

A second example stems from Sarah Moss's (2012) solution to Wittgenstein's 'color exclusion problem' (1929) for his Tractarian project. Wittgenstein wanted a reduction of determinate-determinable talk that would make it more-or-less analytic that everything had exactly one determinate of every determinable. He despaired of the attempt, but Moss came up with an elegant solution.

To illustrate it, suppose there are only four colors: red, blue, yellow, and green. ${ }^{8}$ Moss's solution is to take as basic the properties red-or-yellow and redor-blue. Then to be red is to have them both; to be yellow is to have the first but not the second, to be blue is to have the second but not the first; and to be green is to have neither.

Despite the names, don't think of red-or-yellow and red-or-blue as essentially disjunctive states. We have disjunctive names for them, but the states are categorial color-theoretic states, forming the ultimate bases for the colors themselves. We give them disjunctive names because, in the order of experience, we come to know them by first coming to know the color states that they work together to make up, and since (e.g.) red-or-yellow is a state that helps make up both red and yellow, we find its disjunctive name useful.

Now consider

(7) This patch of color is red or yellow $<$ this patch of color is red.

Given the Mossian reduction, this again looks wrong. The patch's being red is a more complex state, dragging in the red-or-blue property, than the state of being red-or-yellow. A patch's being red is not a way to realize its being red-oryellow, nor is it a 'witness' of its red-or-yellow-ness. (7) suggests that it is the colors themselves which are basic, when the Mossian clearly thinks that it is the properties red-or-yellow and red-or-blue that are basic. So, if the Mossian is right, true disjunctions aren't always grounded in true disjuncts.

Either view provides a counterexample to one of the two most plausible candidates for substantive constraints on grounding. The point isn't that Moss and Argle gave us the truth about colors and holes, falsifying the alleged constraints. (The considerations that led Argle to recant later in the dialogue should probably sour us on the Argelian treatment of holes, too, for instance) The point is that the theories are clearly comprehensible, and if they had been true, the grounding principles would have been false. There may be problems with Argle's or Moss's view, but their failure to obey some alleged grounding principle

\footnotetext{
${ }^{8}$ Of course, there are continuously many colors. Moss's solution needs there to be $2^{\kappa}$ determinates for each determinable; since the continuum is $2^{\aleph_{0}}$, the solution works for each continuumvalued determinable. The smaller color space is better for illustrative purposes, though.
} 
isn't among them. So the alleged constraints aren't the sort of thing that can actually constrain theory. Once the correct metaphysical theory has been figured out we can go back and check whether the principles hold; but we can't lay them down in advance as sacred truths that no theory dare violate.

These examples work by finding states that don't really seem to be quantificational or disjunctive, but that we nonetheless express using quantifiers or disjunctions. This suggests a general recipe for undermining proposed substantial constraints. The constraint will focus on how grounding interacts with some logical expression; to undermine the principle, cook up a metaphysical theory where some uses of the expression intuitively don't express a state with the relevant logical features. It also suggests a response on behalf of grounding's logician: Insist that the constraints are only meant to apply to states that are genuinely existential, or disjunctive, or what-have-you.

Unfortunately, I doubt this reply can be maintained. Recall again the argument above for

(3) If $P$, then $P \wedge P<P$.

Since ' $P$ ' and ' $P \wedge P$ ' express the same non-conjunctive state, it's an instance of a constraint applying to a not-genuinely-conjunctive state. If we accept (CONT), then (3) would seem to be a counterexample to (CONJ); if not, then (CONT) has to go. Either way, some central principles of the 'logic' of ground have to be given up.

A grounding logician may resist this argument by reifying facts and insisting (as I think he should not) that the fact that $P$ is distinct from the fact that $P \wedge P$. In that case, neither (his variant of) (CONT) nor (CONJ) has to be given up, for he can insist that the fact that $P \wedge P$ is 'genuinely' conjunctive. But now I fear the logician has lost the force of his reply to the original examples. Imagine, for instance, that Argle reifies facts. (This would be a very un-Argelian thing to do, but our imaginations will stretch.) He may then go on to insist that there are two distinct facts - the fact that his cheese is perforated, and the fact that there is a hole in his piece of cheese - and to insist that the latter is grounded in the former. He also denies that for some entity $a$, the fact that there is a hole in his piece of cheese is grounded in $a^{\prime}$ s being a hole in his piece of cheese.

Suppose he does all this. What would keep his theory from undermining the logician's principle that existential facts are grounded in their instances? Presumably that the existential fact is somehow not 'really' existential. But in what sense? He cannot say 'in the sense that it is not grounded in its instances', for that would trivialized the principle. But it is hard to see what other reason he could give for denying the fact's 'real' existential-ness that would not also be a reason to deny the 'real' conjunctiveness of the fact that $P \wedge P$.

The grounding logician who responds to Argle's or Moss's theories this way draws himself a very fine line, and I cannot see how he will walk it without falling off. Absent of such a line, though, it looks like we cannot tell, in advance, how realization-grounding will interact with our logical terms. If so, 
then sussing out the 'logic' of grounding a priori, independent of the particular theories that might use the notion, is a fool's errand.

\section{Theoretical Virtues}

Contemporary metaphysics is dominated by a broadly Quinean methodology: Come up with as many theories as you can, and then believe the best one - that is, the most virtuous one, the most elegant and simple and strong one. Each theory has some virtues and some vices, and we look for the theory whose virtues best offset its vices. Ideally, we will find a vice-free theory; more realistically, we will be stuck with a few vices, and so we look for the theory with powerful enough virtues to make those vices worth it. The vices of a virtuous theory are still vices - bad-making features - and the theory would be better if it could lose those vices without sacrificing its virtues.

One strand of enthusiasm for grounding suggests it can help theories do this. The idea is that only the 'fundamental' parts of theories - that is, only the parts of theories that are ungrounded - have to be parsimonious, elegant, simple, and so on. The grounded parts can be as ugly or profligate as we please.

Jonathan Schaffer, for instance, writes

Occam's Razor should only be understood to concern substances: do not multiply basic entities without necessity. There is no problem with the multiplication of derivative entities - they are an "ontological free lunch." (Schaffer 2009: 361)

And Ross Cameron:

Derivative entities don't lack being, but their being is inherited from their grounds, and so... acknowledging that there are these things incurs no further ontological commitment over acknowledging their ultimate grounds. (Cameron 2014: 100)

These authors are focused on the virtue of parsimony, claiming that a theory shouldn't score worse on that dimension just because it has more grounded entities in it. In principle, similar claims could be made on behalf of other theoretical virtues: Complexity or inelegance in the derivative is no mark against a theory's simplicity or elegance. Among those who think that grounded parts of theories are exempt from the theory-choice calculus, egalitarians think that all of the grounded's vices are irrelevant, whereas partisans think that only some of them are.

I suspect that the egalitarian option is the most defensible, but I won't argue for that here. Rather, I'll follow the lead of Schaffer and Cameron in focusing on the particular virtue of parsimony. The claim is that this virtue, at least, is only a virtue insofar as it applies to the ungrounded parts of a theory. When it comes to what is grounded, it makes no difference. 
As already noted, grounding can be understood either as a kind of realization or as a kind of dependence. If 'grounding' means realization-grounding, then I have sympathy for the claim. When we calculate Argle's ontology, we don't include holes. And we do this for the obvious reason that, in some very real sense Argle thinks there aren't holes - even though he assents to 'there is a hole in my cheese.' Hole talk is, for Argle, just talk; and its being just talk shows up in the way existential claims about holes are grounded in entirely hole-free states.

It will take some work to say precisely what kind of realization-grounding claims protect parts of a theory from Occam's Razor. Argle's holes are surely protected. On the other hand, the mere fact the existence of a philosopher is realization-grounded in (inter alia) my being a philosopher doesn't mean that philosophers generally are exempt from Occam's Razor. The rough form of the idea, presumably, is that a theory which says that there are Fs need not count as less parsimonious for saying that there are Fs insofar as, according to the theory, there being $F \mathrm{~s}$ is realization-grounded in truths that don't involve things which are in fact $F$. The rough form needs polishing up, and I will leave that to those who think realization-grounding is the best tool for the job.

In the quotes above, however, neither Schaffer nor Cameron are best understood as talking about realization-grounding. ${ }^{9}$ According to them, that which is grounded in that it is ontologically dependent is no mark against a theory's parsimony. I think this claim misguided, and argue for that in the remainder of this section.

\subsection{The 'Being Fluid'}

I guess the intuitive case for grounded entities forming a 'free lunch' is given in the quote by Cameron above: grounded things 'inherit their being' from their grounds, and so add no 'additional being' to the world, but are just the 'same being' passed up from their grounds.

This sort of talk makes me think of things as filled with a special kind of 'being fluid'. Ungrounded things have their own quantity of this fluid, but grounded entities don't. Rather, the grounding relation puts a tube between the derivative and its grounds. The grounds then 'pump' this fluid through the tubes into the grounded things. Since introducing derivative things doesn't put any new fluid into the system (goes the idea), we get the derivative entities for free. ${ }^{10}$

This is just a metaphor, of course; and maybe it's the wrong metaphor, but it's the only one I've found that really gives me any grip on this 'inherited being' talk. But let's not quibble over the metaphor, or ask uncomfortable questions about it. (Like: If the derivative get their being fluid from their grounds, to the

\footnotetext{
9 Cameron (2014: 100 n. 22) explicitly distinguishes his use of 'derivative' from a more deflationary one that would fit better with realization-grounding.

${ }^{10}$ I owe this metaphor to Robbie Williams, in conversation.
} 
grounds lose more fluid and thereby become 'less real' the more they ground?) Even spotting the metaphor, I don't see how we get to any particular claim about theoretical virtues. To think that derivative entities are no cost, we have to think that the being fluid is very, very expensive while containers are cheap. But why think that? Minimization of a certain stuff, the 'being fluid', is supposed to be virtuous, whereas minimization of the number of things that have it is not. But without something more than this shaky metaphor it is hard to see why the virtues of minimization should line up this way.

Of course, even if this is right, it doesn't show that we have reason to reject Schaffer's and Cameron's view, but rather that we lack good reason to endorse it. If I want to give you reason to reject it (and I do), I had better come up with an argument.

\subsection{The Overgeneration Argument}

Here is my argument: If Schaffer and Cameron are right, we cannot explain why certain bad theories are bad. Take any theory $T$ according to which there are sets, grounded in their members. (For the rest of section 5, 'grounded' will mean 'dependence-grounded'.) Then consider another theory just like it, $T^{2}$, which differs only in that it has two kinds of sets. There are two set membership relations, $\epsilon_{1}$ and $\epsilon_{2}$, and two isomorphic copies of the set-theoretic hierarchy. According to $T,\{$ Jason $\}$ is grounded in me, and so on; according to $T^{2},\{\text { Jason }\}_{1}$ and $\{\text { Jason }\}_{2}$ are both grounded in me, and so on.

If ever a theory should be rejected as unparsimonious, $T^{2}$ should be. $T$ is just like $T^{2}$ but for the extra set-theoretic hierarchy, and so should be preferred. But that extra set-theoretic hierarchy is derivative, which is to say, grounded. If the derivative don't count against parsimony, there is no reason to prefer $T$ to $T^{2}-$ a bad result.

An objection: 'Suppose we can't say that $T^{2}$ is more virtuous than $T$. It's still an obviously bad theory. Why can't we just reject it for its obvious badness? We don't have to use theoretical virtues to rule out everything we want to rule out!'

Reply: We can, of course, reject a theory for its obvious badness. And some theories should be ruled out because of some flaw - maybe even some obvious flaw - unrelated to the theoretical virtues. But if a theory does have some obvious flaw, we should at least be able to say what it is. It is very embarrassing to know that a theory ought to be rejected but have no systematic explanation as to why. Our reason for rejecting $T^{2}$ seems pretty plain: It multiplies entities beyond necessity. It is unparsimonious in the extreme, and ought to be rejected. But we can say this only if we admit that the derivative count against parsimony; and no other candidate reason for rejecting $T^{2}$ presents itself.

That, anyway, is my argument. Schaffer (2015: §8) has considered it and offered a reply. ${ }^{11}$ In it, he notes that, in order to get a consistent $T^{2}$, we have to

\footnotetext{
${ }^{11}$ In his version he considers two mereologies, not two set theories, but the issues are substan-
} 
modify the axioms of each set theory a little bit. Otherwise we'll end up with unfortunate Russell's-paradox-style problems. But these axiomatizations make the resulting set theories less elegant. As a result, we can reject $T^{2}$ as being the less elegant theory of the two.

In offering this line of response, Schaffer reveals himself as a partisan: The set-theoretic axioms of $T$ and $T^{2}$ are all grounded, and an ecumenical approach would exempt them from the demands of simplicity. But even granting Schaffer his partisanship, I don't think the response works. Granted, we must modify $T^{2}$ 's axioms to get our two set-theoretic hierarchies. But the modifications aren't very severe.

Suppose that $T$ contains an axiomatization of Zermelo-Frankel set theory. Since $T$ contains non-sets, it will need a predicate 'set' and a membership relation ' $\in$ ' for the axiomatization, and some (but not all) of the quantifiers in the axioms will have to be explicitly restricted to sets. To get $T^{2}$, we add ' 1 ' subscripts to our original set and membership predicates, add predicates 'set ${ }_{2}$ ' and ' $\epsilon_{2}$ ', and copy the axioms over. Then we do one final thing: Whenever we had unrestricted quantification in the original axioms, the quantifications in the axioms for sets ${ }_{2}$ are restricted to things that are not sets ${ }_{1}$, and the quantifications in the axioms for sets $s_{1}$ are restricted to things that are not sets ${ }_{2} .{ }^{12}$ This appies to the replacement and comprehension schemas as well: whatever formulas are plugged into those schemas for sets ${ }_{1}$ have to have quantifiers restricted to things that are not sets 2 , and vice versa. Once we have done that, everything works out fine.

Are these axioms more ungainly than those of $T$ ? Sure, a little bit; restricting quantifiers is always a bit ugly. Are they significantly uglier? Not obviously. Quantifier restrictions are a pretty simple deal. $T$ might edge out $T^{2}$ by a bit, but it's not by much.

We're familiar with theory-pairs of these sorts — pairs where one is a little bit more complex than the other, without being outrageously so. The Newtonian theory of spacetime posits objective facts about whether two points at different times count as the 'same place' or not. The Galilean theory is just like the Newtonian minus this same-place-across-time relation. The simpler Galilean is clearly more virtuous, and to be preferred over the Newtonian. But the Newtonian isn't a non-starter. It's a good theory, beaten by a better one.

$T^{2}$ isn't like the Newtonian theory. It's not a pretty good theory beaten out by a better, simpler one. It really is a non-starter. We need to explain why this is so. Simply pointing out that $T^{2}$ is a bit uglier than $T$ isn't enough.

There is more to Schaffer's response than this, though. Notice that, in $T$, all the non-sets get to be in sets, while in $T^{2}$ only the non-sets 2 get to be in sets 1 , and vice versa. As a result, $T^{2}$ 's two set-theoretical hierarchies aren't maximally permissive: they're not making sets $s_{i}$ out of all the things available to make sets ${ }_{i}$ out

\footnotetext{
tially the same.

${ }^{12}$ With one exception; each theory's empty set ${ }_{i}$ axiom should say that there is a set $_{i}$ with nothing whatsoever - quantifiers wide open - in it.
} 
of. Schaffer suggests that this is a pretty big vice - a lot bigger than a few extra quantifier restrictions. It undercuts part of the initial motivation for set theory: not all the entities are available to form sets. If we try to fix this by modifying the axioms differently, so as to allow sets ${ }_{1}$ with sets $s_{2}$ as members, and vice versa, things get sticky. We will have to give up on the extensionality of sets, which was another part of their raison d'être. Set theory as it stands is, as Schaffer puts it, 'maximally permissive, given the demands of [its] associated extensionality principles' (2015: 657). So is classical extensional mereology, which is why a similar type of example using what Schaffer calls a 'doubled-mereology' won't work, either. In essence, Schaffer suggests a kind of special-purpose virtue suitable at least for evaluating set-theories and mereologies - of maximal extensionality. The idea is that violating maximal extensionality is a pretty big vice, and that, rather than anything about parsimony, is why we find $T^{2}$ a non-starter.

Unfortunately, I don't think maximal extensionality can do the kind of work Schaffer wants it to. If violating maximal extensionality really is a huge vice, then no virtuous theory can include both extensional mereology and set theory. Unrestricted extensional mereology and unrestricted extensional set theory are mutually inconsistent (see Hudson 2006). If we want extensional mereology and extensional set theory, then we will either have to restrict composition, so that some things do not compose a whole, or restrict set theory, so that some things do not have singletons.

There are various natural ways to do this. We might restrict set theory so that everything save some 'proper classes' have singletons. ${ }^{13}$ We might instead restrict mereology so that only non-sets compose wholes. These restrictions would be unfortunate, but theories that made them would not seem to be vicious enough to warrant rejection. The restriction of mereology to non-sets, for instance, seems fairly natural. Mereology would be restricted, but in a way that followed a deep division in reality - the division between sets and non-sets, or between concrete and abstracta. A small price to pay for consistency.

If we can restrict mereology to make it consistent with set theory, or restrict set theory to make it consistent with mereology, then violating maximal extensionality isn't such a huge vice after all. And if it isn't a huge vice, then we

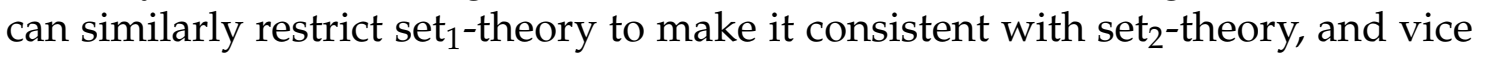
versa. And there is a perfectly good rationale for $T^{2}$ 's restrictions. We can think of the $\operatorname{set}_{1} \mathrm{~s}$ as what we get when we start with a particular ontological category - the category of not-any-sort-of-set - and applying the standard iterative process lying behind the iterative conception of set (Boolos 1971). We can think something similar about set ${ }_{2}$-theory. The restrictions on the set $_{i}$-theory axioms are required by consistency and perfectly well-motivated on their own, just as restrictions on mereology or ordinary set-theory are in theories with both. When it comes to maximal extensionality, $T^{2}$ seems to do just as well as a virtuous mereology-plus-set-theory. But $T^{2}$ still strikes us as a non-starter, so it

\footnotetext{
${ }^{13}$ This is what Lewis (1991), who endorses both extensional mereology and extensional set theory, opts for.
} 
must be for some reason other than its failure at maximal extensionality.

The problem, of course - as should be blindingly obvious - is that one of $T^{2}$ s hierarchies is redundant. We have a bunch of entities doing the same job twice. But it's very hard to say that without admitting that we ought not to multiply sets - derivative entities, in this case - beyond necessity. (For what is a redundant entity if not an entity beyond necessity?) The obvious explanation is the right one. But if so, parsimony applies to the derivative, too. ${ }^{14}$

\subsection{Undercutting the Case for Fundamentalism}

I have argued that fundamentalism is false. Schaffer (2015) has recently argued at length that it is true. If my argument is correct (as I think it is), then his is not. You may fairly wonder where I think he goes wrong. If so, then you're in luck; I'm about to tell you.

\subsubsection{The Analogy with Conceptual Parsimony}

One of Schaffer's arguments (2015: §4) is by analogy. We think that conceptual, or ideological, parsimony is a virtue: Theories are better when they make do with fewer notions. But this only applies to a theory's fundamental notions - its basic ideological building-blocks. And what goes for ideology goes for ontology, too: If conceptual or ideological parsimony only tells us to cut down the number of fundamental notions, then ontological parsimony should only tell us to cut down the number of fundamental entities.

I think this argument nicely illustrates the dangers, outlined in §3, of assuming fundamentality talk should be uniformly understood as a specific kind of ungroundedness. Schaffer begins with a compelling case that only 'fundamental' notions count against a theory's ideological parsimony. By analogy, he concludes that only 'fundamental' entities count against a theory's ontological parsimony. By 'fundamental entities' he explicitly means entities that are not grounded, and his understanding of 'grounded' fits best with what I have called 'dependence-grounding'. So his analogy is meant to support

(8) When evaluating a theory, only entities which are not dependence-grounded in something else count against its ontological parsimony.

Unfortunately, his case that only 'fundamental' notions count against ideological parsimony only works because he taps into a different notion of 'funda-

\footnotetext{
${ }^{14}$ In personal communication, Cameron suggested that this doesn't quite undermine his view, for he can insist that there is a general virtue of avoiding epiphenomena (which applies across the board) and a specific virtue of minimizing entities (which applies only to the 'fundamental,' that is, ungrounded.) $T^{2}$ is bad for violating the first, and the second has nothing to do with it. The double-virtue view strikes me as unmotivated, but as a backup reply note that, on the proposed view, when we get to the theories discussed in the next section, Gerald's is to be preferred to Esthers, which strikes me as obviously false.
} 
mental'. Once we unpack that notion, we see that (8) isn't analogous to what Schaffer's ideological case establishes.

What is his ideological case? Start from the observation that in classical extensional mereology, 'parthood' and 'overlap' are interdefineable: $x$ is a part of $y$ if and only if everything overlapped by $x$ is overlapped by $y$, and $x$ overlaps $y$ if and only if they share a part. ${ }^{15}$ But a theory isn't worse off for having both of these in it rather than just one. Schaffer writes,

Imagine that one has just started to theorize about mereology, with [parthood] as one's lone primitive notion. One then sees how to define overlap. It would be deeply confused to think, 'Alas! I have suffered a setback! The conceptual economy of my theory has been compromised!' (Schaffer 2015: 650)

Since 'overlap' is defined in terms of 'parthood', it is not fundamental, and since it is not fundamental, it is an ideological 'free lunch': it adds no conceptual complexity to a theory.

I have no beef with Schaffer's ideological case. If one notion can be defined in terms of another, then counting both notions against a theory's conceptual economy would be double-counting. But I don't see that it gives any support to (8). 'Overlap' is not fundamental in the imagined theory insofar as it is defined. When theorizing, we often take some notions as basic - as 'primitive', given no further analysis. We then use them to define other notions. Sometimes, we call the primitive notions 'fundamental' and the other notions 'derivative'. But the relationship between primitive and fundamental notions isn't relevantly like the relation between a thing and its dependence-grounds. If 'overlap' is defined in terms of 'parthood', then overlap just is part-sharing, and 'overlap'using sentences express the same states, using different terminology, as their 'parthood'-using analysans. We might fairly think this a species of realizationgrounding, but it is nothing at all like the grounding relation that is meant to hold between a singleton and the element on which it depends.

In other words, Schaffer's case supports the analogy with (8) only if it supports

(9) When evaluating a theory, only notions which are not dependence-grounded in something else count against its conceptual parsimony.

But it doesn't, because the relationship between a defined term and its definiens is not at all like that of dependence-grounding.

And (9) seems wrong on its own merits. Consider a classical theory of propositions, according to which they are a special kind of representational entity that 'tracks' the truth. The imagined theory (inspired by van Inwagen 1986: 202) accepts all (non-pathological) instances of

\footnotetext{
${ }^{15}$ Schaffer's original example uses proper parthood rather than parthood. I've changed it only because it makes the definitions simpler.
} 
(T) $\ulcorner$ If $\phi$, then the proposition that $\phi$ is true $\longleftarrow \phi\urcorner$.

Furthermore, all other truths using 'true', as applied to propositions, are somehow dependence-grounded in ways that lead back to (T). Thus the notion of propositional truth is, always and everywhere, dependence-grounded; a complete description of the dependence-fundamental will never need to use the notion. Yet the term lacks any definition in terms of anything more basic. More tellingly, though, there is little reason to think the notion does not count against the theory's conceptual parsimony. If we could keep the theory but somehow eliminate or define away the notion, we would end up with a better theory. This suggests that the mere dependence-grounding of the notion doesn't let it off the parsimony hook, souring us on (9). But absent any good reason to accept (9) we have no analogy to get us to (8).

\subsubsection{The Direct Argument}

Schaffer's other argument is direct. He asks us to consider two theorists, Esther and Feng:

Esther posits a fundamental theory with 100 types of fundamental particle. Her theory is predictively excellent and is adopted by the scientific community. Then Feng comes along and - in a moment of genius - builds on Esther's work to discover a deeper fundamental theory with 10 types of fundamental string, which in varying combinations make up Esther's 100 types of particle. This is intended to be a paradigm case of scientific progress in which a deeper, more unified, and more elegant theory ought to replace a shallower, less unified, and less elegant theory. Feng's theory is evidently better in every relevant methodological respect. (Schaffer 2015: 648)

Schaffer goes on to point out that, if we pay equal attention to fundamental and derivative ontology, Feng's view should do worse in terms of parsimony. After all, 110 is bigger than 100! Since his view is 'better in every relevant methodological respect,' we shouldn't pay equal attention to fundamental (that is, ungrounded) and derivative (that is, grounded) ontology.

It's not enough to simply agree with Schaffer that Feng's view should be preferred to Esther's. After all, Feng's view might be an overall better theory than Esther's despite being less virtuous in the parsimony department. Theory choice is a holistic weighing and balancing of virtues, and the winning theory may have some vices of its own. This, presumably, is why Schaffer claims that Feng's theory is 'evidently better in every methodological respect' [my emphasis]; unless that is true, the case shows nothing about whether ungrounded entities count against a theory's parsimony.

But before we consider whether this stronger claim is true, let's step back and ask ourselves whether Feng's theory really ought to be preferred to Esther's. The case, as it stands, is under-described. Knowing just what to say about the 
case depends on what it means to say that Feng's ten types of string 'in varying combinations make up Esther's 100 types of particles'. There are a couple of dimensions to consider.

First, the nomic dimension. We already know that Esther's 100 types of particles obey certain laws. Call these the E-laws. Since there are 100 types of particle, there will be a lot of E-laws, and a lot of variables in those laws. What happens when Feng posits his ten types of string?

Here's one way it could go. Along with the strings, Feng may also posit 'F-laws' for how those strings behave, both on their own and in concert. Further, when we couple these laws with the 'recipes' for making particles from strings, most or all of the E-laws fall out as consequences. If so, then Feng's theory is stronger than Esther's: Feng uses a much smaller group of laws, combined with his fewer kinds of things, to guarantee all the behavior that would otherwise have needed Esther's many, many E-laws. Call this the systematizing interpretation.

Here is another way it could go. Each type of particle may be made up of some strings, but there may be no principles whatsoever that tell us anything about the behavior of strings outside particles. It might be that (i) strings never exist without being part of a particle, and (ii) there are no interesting generalizations whatsoever about how particles made up out of the same strings, or the same number of strings, or isomorphic combinations of strings, or whatever, behave. A particle's string-composition may give us by itself no useful information at all about how that particle will behave. Call this the unsystematic interpretation.

Enough of the first dimension. The second is conceptual. Esther's theories will presumably have predicates for each of the different types of particle. What happens to these predicates in Feng's theory?

It may be that these hundred predicates are definable in terms of ten predicates for strings, perhaps in conjunction with some supplementary predicates (like 'is a part of'). Call this the conceptually parsimonious interpretation. But maybe no such definition is possible. The relationship between the strings and the particles may not be systematic enough to allow such definitions. Call this the conceptually profligate interpretation.

Our natural reaction to Schaffer's story is to find Feng's theory obviously preferable to Esther's. But that, I submit, is because we naturally read Schaffer's story in the systematizing and conceptually parsimonious way. On this interpretation Feng's theory has the virtues of unification and systematization by reducing all the E-laws to a handful of F-laws, and the virtues of conceptual parsimony discussed in the last section. Those are some pretty powerful virtues, especially since the reductions give us a tenfold decrease in complexity.

This, by itself, doesn't tell us whether, if this is what Feng's theory is like, it is more ontologically parsimonious (as Schaffer would have it) than Esther's is; it only tells us that Feng's theory has a lot of powerful virtues. Schaffer holds that Feng's theory has no vices whatsoever; in fact, Schaffer holds that Feng's the- 
ory is more parsimonious than Esther's, since ten is smaller than 100, and only ungrounded entities count. The alternative is that Feng's theory is a little less parsimonious than Esther's, but the small sin against ontology is outweighed by the tremendous gain in conceptual parsimony and systemization.

How do we adjudicate these options? Well, if Schaffer is right, then when we consider his story under an unsystematic and profligate interpretation, Feng's theory should still come out triumphant, because ten is still smaller than 100 . Unfortunately, Feng's story doesn't naturally lend itself to such an interpretation. To come up with one, let's start with a warm-up exercise.

Consider Gerald. Before Feng came along, Gerald attempted to offer a unification of Esther's theory. According to Gerald's theory, there is only one fundamental type of thing - something he calls 'coffee cups'. (They aren't actual coffee-cups, but coffee-cup-shaped subatomic entities.) According to Gerald, there are exactly two coffee cups in existence, one on top of the other. The only free variable governing the coffee cups is the angle between their handles. (And when the angle changes, it doesn't have to move continuously; it can 'jump' from one angle to another without passing through the intervening ones.)

Esther's theory allowed for continuum-many particle distributions (let us suppose). Gerald noted that there were continuum-many angles that could have separated the cup-handles. So he introduced a correspondence, $f$, between cup-handle-angles and distributions of particles, and then insisted that how the world was, particle-wise, was grounded in the cup-handle-angle. More precisely, Gerald's theory included the following grounding-schema:

(10) The world is in state $f(n) \longleftarrow$ the angle between the cup-handles is $n$.

Assuming (as seems reasonable) that every truth about the particles can be grounded in the world being in its respective state $f(n)$, the only ungrounded truths on Gerald's theory are ones that involve the coffee-cups and the angle between their handles.

Gerald's theory is neither systematic nor conceptually parsimonious. There are no laws of cup-handle-evolution that explain the E-laws, and there is no way to define any particle-predicates in terms of cup-handle-angles. But it is a theory according to which there are fewer fundamental things (only one kind, and two instances of it). And it seems eminently obvious that Gerald's theory makes no gains over Esther's.

Thus ends the warm-up exercise. We can interpret Schaffer's story as unsystematic and conceptually profligate simply by assuming Feng's theory relevantly similar to Gerald's. Having posited his ten kinds of string, Feng could have introduced some map between some feature of the strings and global particle-states that would have allowed him to pull the same trick. On an unsystematic and profligate interpretation of Schaffer's story, that is just what Feng did. But clearly, if Feng's theory is like this it is no improvement over Esther's.

If Schaffer is right, then Feng's theory, on this interpretation, is better than Esther's, because he has ten kinds of ungrounded entities to her 100, and ten is 
fewer than 100. By the same token, if Schaffer is right, then Gerald's theory is better than both of theirs, since he has only one kind of ungrounded entity and one is fewer than both ten and 100. Clearly, though, Gerald's theory is no better than these other two (and quite probably worse). So Schaffer must not be right after all.

In light of all this, the best explanation for our natural reaction to Schaffer's story is that we interpret it in the systematizing and conceptually parsimonious way, we take the extra ten kinds of entities as a small mark against Feng's theory, but we find the price well worth paying for the tremendous gains in systematicity and conceptual parsimony that it allows. Feng's theory may be superior, but it is not superior in every methodological respect after all.

\section{Shifting Debates}

The temptation of those with hammers to see only nails is an all too human one. And grounding can present itself as a very appealing hammer indeed. Several authors have been seduced into seeing any number of venerable debates in grounding-theoretic terms. For instance, we have Schaffer:

... [in] the debate over universals, both the realist and the nominalist accept the existence of general properties. The dispute is over whether properties are fundamental, or whether they are derivative...

... [the] debate over the mind is not a dispute over whether mind or matter exist, but rather over whether mind is based in matter. The debate... over substantival space is not a dispute over whether there is space, but rather whether space is grounded in its occupants...

... a meta-metaphysics that would make sense of these central questions must make sense of claims of grounding. These central metaphysical questions are not questions about whether entities exist, but only how they do. (2009: 362-363)

Following suit, Dasgupta (2011 and 2015) interprets relationalism as the doctrine that regions of space(-time) are grounded in spatial (or spatiotemporal) relations, and physicalism as the doctrine that mental facts are grounded in physical facts.

I'm inclined to think that these tendancies are a mistake no matter what we mean by 'grounding'. But I must confess that, if we mean 'realizationgrounding', the error is less obvious, and I cannot quite see how the argument will go. When we mean 'dependence-grounding', though - as Schaffer, for instance, explicitly does - the mistake is serious. Exactly what the mistake is, however, depends on how we interpret Schaffer's quote above, for it admits of multiple interpretations. 
To see these interpretations, focus on 'the debate over universals', where the interested parties are 'the realist and the nominalist'. The realist is an easilyrecognized fellow; he believes that there are universals, which are a sui generis entity of a special kind with a special role to play. ${ }^{16}$ But who is this nominalist? Someone who accepts the existence of 'general properties'. Would Joseph Melia count, then? For he "defend[s] an austere version of nominalism, one that rejects classes, universals, tropes, states of affairs and other such metaphysical extravagances... [T] he world contains concrete particulars, and only concrete particulars" (Melia 2015: 175). This certainly doesn't sound like someone who accepts the existence of 'general properties'.

If Melia is indeed supposed to be one of these nominalists, then Schaffer accuses him of misunderstanding his own view. If he is not, then Schaffer's taxonomy implicitly cuts him out of the dialogue. Either way, there is a viable position on the table - the position that there are no general properties, a position that some feel inclined to defend - that is getting passed over in silence. This is a bad thing. We cannot make theories we dislike (and the arguments for them) go away by simply choosing to ignore them. If grounding tempts us to do this, then grounding is a devious little devil indeed.

I do think that some authors in the literature really have thought that debates which seem to be about what exists have really been about what is grounded all along, and have illicitly shifted their view of the issues to follow suit. This is a bad thing; in fact, it strikes me as so obviously a bad thing that I don't really know how to argue further that we shouldn't do it. Whatever we think about grounding, we cannot be seduced into ignoring other important metaphysical issues.

But, in fairness to Schaffer, I do not think he is making this mistake. I think he intends his use of 'nominalism' to be understood in a way that excludes Melia, and that he thinks he has good reasons for the exclusion. My interpretation is based on how he treats another debate, one in the philosophy of mathematics. There he considers a debate between constructivists and 'realists', and characterizes that debate as one about grounding (2009: 362). Conspicuously absent in his caracterization is, of course, the self-proclaimed eliminativist who, like Hartry Field $(1980,1989)$, explicitly rejects the existence of numbers.

A bit later, Schaffer concedes that "There is no problem making room for existence questions on [my] view," but then goes on to say:

That said, I also doubt that there are many important metaphysical existence questions. Or at least I would maintain that the usual candidates (e.g. the question of whether numbers exist) fail, and would ask [those who think otherwise] for better examples. (363)

If I understand him right, Schaffer's position is this: There may be debates

\footnotetext{
${ }^{16}$ Or does he? I would have expected D. M. Armstrong $(1978,1997)$ to be a paradigm realist; yet he seems open to the idea that universals are 'abstractions' from facts. On this taxonomy, wouldn't that make him, surprisingly, a nominalist?
} 
about what exist, but they aren't very interesting. The people who say there aren't certain kinds of things are just obviously wrong. So we ought to stop having those debates and start instead having the genuinely interesting ones, that is, ones about grounding. Hammer, meet nail.

So what do I think Schaffer's mistake is, then? Underestimating the power of arguments against certain sorts of entities, say I. He urges me to find a better example of an interesting existence debate than the one about numbers. I refuse; I think the mathematical one is perfect, as it illustrates why the temptation to view everything in grounding-theoretic terms is a dangerous one.

First, the dialectic. The nominalist says, 'There are no numbers!' The platonist points out that numbers seem indispensable in science. The nominalist then trots out Hartry Field's Science Without Numbers project as a way to answer the indispensability argument. At this point, Schaffer responds,

If Field's construction works, for instance, I say it shows how numbers do exist in a world of concrete substances, as grounded in certain features of such substances (e.g., betweenness and congruence relations between substantival spacetime points). This is a better interpretation of the Field construction than Field's own Quinean eliminativist interpretation [according to which there are no numbers], because it reconciles Field's view with the obvious fact that there are prime numbers." (360)

So the battle lines are drawn. We have Field's construction; he says it shows us that numbers aren't needed, whereas Schaffer says it shows us how numbers are grounded in the concrete realm. Schaffer says his interpretation is better. I say it isn't. And if it isn't, then Field's eliminativism about numbers really is an interesting metaphysical view, worthy of discussion rather than consignment to the dustbin of not-about-grounding-and-therefore-not-interesting.

What is wrong with Schaffer's interpretation? I'm inclined to think a handful of things, but I'll focus on the one that I take most central to Field's own motivations. Field's arguments against the existence of numbers are largely epistemic. He worries that, if there is a realm of mathematical entities, then (a) mathematicians know an awful lot about this realm, and (b) their knowledge is entirely mysterious. (Field 1989: 25-30) If there are no such entities, then whatever mathematicians know, it isn't a bunch of facts about some abstract entities, and the mystery is resolved. ${ }^{17}$

How is the mystery resolved if numbers are grounded? The entities are still there; they're still abstract; mathematicians still end up being very reliable about them; and we still have no good explanation for their reliability. Field's interpretation solves Field's problem; Schaffer's interpretation doesn't.

Perhaps that is too quick. Considering epistemic worries, Schaffer suggests that 'if numbers are indeed grounded in the concrete realm, then... they may

\footnotetext{
${ }^{17}$ Well, resolving the mystery takes a bit more work; see Field 1984.
} 
be known via their concrete grounds' (p. 361). But I really don't see any help here. First, I'm not even sure what the proposal is. Is the proposal that, since Field has shown us how the mathematical claims of physics can be licensed by a world with a certain structure, we can come to know claims of pure mathematics by learning about this structure and then abstracting the physical part away? Like, can I learn that the number two is prime by learning some fancy physics involving the number two and primeness and then just taking out the physics bit?

Even if that were right, it would be no help on Field's problem. If there are numbers, then mathematicians already know a lot about them. And they didn't learn this stuff by learning a bunch of physics first. Their knowledge remains mysterious, even if the grounding story could provide an alternative, not-actually-travelled, route to this knowledge.

Second, I don't see why it would be right. Consider a very simple case: Me and the sets grounded in me. If standard set theory is right (and if sets are grounded in their elements), I end up being the ultimate grounds for $\{$ Jason\}, $\{\{$ Jason $\}\},\{J a s o n,\{J a s o n\}\}$, and (in fact) a copy of the whole set-theoretic hierarchy. So a whole load of sets get grounded in me. But even though I know a lot about myself, I don't see any special insight into sets that I get from grounding a bunch of them.

Call a set a me-set iff it is ultimately grounded in me. ${ }^{18}$ Is there a countable me-set $M$ ? If there is, does it have a power-me-set $P$ ? If it does, is there a me-set $S$ that is strictly larger than $M$ but smaller than $P$ ? I'm pretty sure I cannot know the third, given that it's equivalent to the continuum hypothesis. If I can't know that, then what gives me any epistemic traction on the first and second? But the first and second follow from standard axioms of set theory, and so are supposed to be the kinds of things mathematicians get to know unobjectionably.

Here, I know about as much about the ultimate grounds of some entities that one could reasonably hope to know, and remain completely stumped as to how this could give me any knowledge of the things it grounds. And if knowledge of the concrete grounds of sets doesn't give us any epistemic purchase on the sets, knowledge of a Field-style concrete ground for numbers won't give us any purchase on them, either. How could it?

One suspects the answer is supposed to be, 'because it's grounding.' But grounding isn't magic. We can't make genuine, difficult philosophical problems go away by pointing our wands in the right direction and uttering the incantation, 'Let it be grounded!' These philosophical problems are hard - so hard that some have thought the right solution is to deny the apparently obvious and insist that there are no numbers (or properties, or whatever) after all. We do ourselves no favors by ignoring theories that attempt to solve them and then pretending there was no problem to be solved in the first place.

\footnotetext{
${ }^{18}$ Better: If it is a set-theoretic construction purely out of $\{$ Jason $\}$.
} 


\section{CONClusion}

I won't lie: Grounding is a cute little number, and some philosophical positions wear it well. But it's starting to oversaturate the market. Its advertising campaign tells us it can be outfitted with a solid logic, that it can block swipes from Occam's razor, and that it covers any philosophical position you care to name. In truth, it is a much more limited accessory. We can use it to define a type of fundamentality, sure; but there are other, equally legitimate uses of 'fundamental' that won't be so-defined. There might be some general logic-like principles that govern it, they won't be the sort of things that we can lay down with a priori certainty. But it doesn't relieve its wearer from the call to virtue, and, despite the zeal of its marketers, it does not go with every philosophical position. Let us keep it for when it is useful, of course. But in our enthusiasm for this new rage, let us not forget the older, hardier parts of our philosophical wardrobe that have served us so well for so long.

\section{REFERENCES}

Armstrong, David M. (1978). A Theory of Universals, volume 2 of Universals and Scientific Realism. Cambridge University Press.

— (1997). A World of States of Affairs. Cambridge: Cambridge University Press.

Audi, Paul (2012). "A Clarification and Defense of the Notion of Grounding." In Correia and Schnieder (2012), 101-121.

Barnes, Elizabeth (2012). “Emergence and Fundamentality." Mind 121: 873-901.

— (forthcoming). "Symmetric Dependence." In Ricky Bliss and Graham Priest (eds.), Reality and its Structure. Oxford Univerisity Press.

Boolos, George (1971). "The Iterative Conception of Set." The Journal of Philosophy 68(8): 215-231.

Cameron, Ross P. (2014). "Parts Generate the Whole but are Not Identical to It." In Donald Baxter and Aaron Cotnoir (eds.), Composition as Identity, 90-107. Oxford University Press.

— (2015). The Moving Spotlight. Oxford: Oxford University Press.

Chalmers, David, David Manley and Ryan Wasserman (eds.) (2009). Metametaphysics. Oxford: Oxford University Press.

Correia, Fabrice (2010). "Grounding and Truth-Functions." Logique et Analyse 53(211): 251-279. 
Correia, Fabrice and Benjamin Sebastian Schnieder (eds.) (2012). Metaphysical Grounding: Understanding the Structure of Reality. Cambridge: Cambridge University Press.

Daly, Chris (2012). "Scepticism about Grounding." In Correia and Schnieder (2012), 81-100.

Dasgupta, Shamik (2011). “The Bare Necessities." Philosophical Perspectives 25: 116-160.

— (2014). “On the Plurality of Grounds." Philosophers' Imprint 14(20): 1-28.

— (2015). "The Possibility of Physicalism." The Journal of Philosophy 111(9/10): 557-592.

deRosset, Louis (2014). “On Weak Ground." The Review of Symbolic Logic 7(4): 713-744.

Field, Hartry (1980). Science Without Numbers. Malden, Mass.: Blackwell.

- (1984). "Is Mathematical Knowledge Just Logical Knowledge?" The Philosophical Review 93(4): 509-552. Reprinted with postscripts in Field 1989.

- (1989). Realism, Mathematics, and Modality. Blackwell.

Fine, Kit (2009). “The Pure Logic of Ground." The Review of Symbolic Logic 5(1): $1-25$.

— (2012). "Guide to Ground." In Correia and Schnieder (2012), 122-138.

Hofweber, Thomas (2009). "Ambitious, Yet Modest, Metaphysics." In Chalmers et al. (2009), 260-289.

Hudson, Hud (2006). "Confining Composition." The Journal of Philosophy 103(12): 631-651.

Koslicki, Kathrin (2015). "The Coarse-Grainedness of Grounding." In Karen Bennett and Dean W. Zimmerman (eds.), Oxford Studies in Metaphysics, volume 9, 306-345. Oxford: Oxford University Press.

Lewis, David (1983a). "New Work for a Theory of Universals." The Australasian Journal of Philosophy 61: 343-377. Reprinted in Lewis 1999: 8-55.

— (1983b). Philosophical Papers, volume 1. Oxford: Oxford University Press.

- (1991). Parts of Classes. Malden, Mass.: Blackwell.

- (1999). Papers in Metaphysics and Epistemology. Cambridge: Cambridge University Press. 
Lewis, David and Stephanie Lewis (1970). "Holes." The Australasian Journal of Philosophy 48: 206-212. Reprinted in Lewis 1983b: 3-9.

Litland, Jon Erling (2015). "Grounding, Explanation, and the Limit of Internality." Philosophical Review 124(4): 481-532.

Melia, Joseph (2015). "Nominalism, Naturalism, and Natural Properties." In Ghislain Guigon and Gonzalo Rodriguez-Pereyra (eds.), Nominalism about Properties: New Essays, 175-188. New York: Routledge.

Moss, Sarah (2012). "Solving the Color Incompatibility Problem." Journal of Philosophical Logic 41: 841-851.

Rosen, Gideon (2010). "Metaphysical Dependence: Grounding and Reduction." In Bob Hale and Aviv Hoffman (eds.), Modality: Metaphysics, Logic, and Epistemology, 109-135. Oxford: Oxford University Press.

Schaffer, Jonathan (2009). “On What Grounds What.” In Chalmers et al. (2009).

- (2015). "What not to Multiply without Necessity." The Australasian Journal of Philosophy 93(4): 644-664.

Turner, Jason (2013). “Existence and Many-One Identity.” The Philosophical Quarterly 63(251): 313-329.

van Inwagen, Peter (1986). "Two Concepts of Possible Worlds." In Peter A. French, Theodore E. Uehling and Howard K. Wettstein (eds.), Midwest Studies in Philosophy, volume XI, 185-213. Minneapolis, Minn.: University of Minnesota Press. Reprinted in van Inwagen 2001: 206-242.

- (2001). Ontololgy, Identity, and Modality. Cambridge: Cambridge University Press.

Wilson, Jessica (2012). "Fundamental Determinables." Philosophers' Imprint 12(4): 1-17.

— (2014). "No Work for a Theory of Grounding." Inquiry 57(5/6): 535-579.

Wittgenstein, Ludwig (1921). Tractatus Logico-Philosophicus. London: Routledge \& Kegan Paul. Translated 1961 by D. F. Pears and B. F. McGuinness.

- (1929). "Some Remarks on Logical Form." Proceedings of the Aristotelian Society Supplimentary Volume: 162-171. 